Research Paper

\title{
MMP9 and IGFBPI Regulate Tumor Immune and Drive Tumor Progression in Clear Cell Renal Cell Carcinoma
}

Tianbo $\mathrm{Xu}^{1 *}$, Su Gao ${ }^{2,3^{*}}$, Jingchong Liu $^{1 *}$, Yu Huang ${ }^{1}$, Ke Chen ${ }^{1}$, Xiaoping Zhang ${ }^{1 凶}$

1. Department of Urology, Union Hospital, Tongji Medical College, Huazhong University of Science and Technology, 1277 JieFang Avenue, Wuhan 430022 , China.

2. Department of Geriatrics, Union Hospital, Tongji Medical College, Huazhong University of Science and Technology, 1277 JieFang Avenue, Wuhan 430022 , China.

3. Institute of Gerontology, Union Hospital, Tongji Medical College, Huazhong University of Science and Technology, 1277 JieFang Avenue, Wuhan 430022 , China.

* These authors contributed equally to this work.

$\square$ Corresponding author: Xiaoping Zhang, Department of Urology, Union Hospital, Tongji Medical College, Huazhong University of Science and Technology, 1277 Jiefang Avenue, Wuhan, 430022, Hubei Province, China. E-mail: xzhang@hust.edu.cn.

(c) The author(s). This is an open access article distributed under the terms of the Creative Commons Attribution License (https://creativecommons.org/licenses/by/4.0/). See http:/ /ivyspring.com/terms for full terms and conditions.

Received: 2020.05.25; Accepted: 2021.01.13; Published: 2021.02.22

\begin{abstract}
Immunotherapy is a novel approach and has been used in various diseases, especially in cancers. Recently, immunotherapy has gradually been used to treat advanced clear cell renal cell carcinoma (ccRCC) or metastatic ccRCC. However, the efficacy of immunotherapy is not satisfying due to the influence of the tumor microenvironment. In this study, we mainly focused on the abundance and function of tumor-infiltrating immune cells (TIICs). Monocyte and TNM stage were identified as independent prognostic factors via CIBERSORT and Cox regression analysis. Then, $c \subset R C C$ patients were divided into high risk/TNMhighMonocyteslow cluster and low risk/TNMlowMonocyteshigh cluster. Further differential gene analysis, protein-protein interaction (PPI) network, and survival analysis screened nine hub genes between the above two clusters. MMP9 and IGFBPI were selected for further study through sample validation. Moreover, gene set enrichment analysis revealed that MMP9 and IGFBPI were involved in tumor immune via mediating cell surface receptor signal pathway, cytokine production pathway, or monocyte signal pathway. In conclusion, these findings suggested that monocyte acted as a protective factor and MMP9/IGFBPI played a vital role in tumor immune, which might become potential novel biomarkers and therapeutic targets for immunotherapy in ccRCC.
\end{abstract}

Key words: clear cell renal cell carcinoma; MMP9; IGFBP1; biomarker; tumor-infiltrating immune cells

\section{Introduction}

Renal cell carcinoma (RCC) is one of the most common solid tumors and makes up about $2-3 \%$ of all adult malignancies [1]. In 2019, approximately 73,000 new RCC patients are diagnosed and more than 14,700 patients will die due to RCC in the USA [2]. RCC encompasses more than 10 different histological subtypes. Clear cell renal cell carcinoma (ccRCC) is the most common subtype of RCC, which accounts for $70-80 \%$ of RCC cases [3,4]. For localized ccRCC, surgery is the most effective treatment and can significantly prolong the survival of patients $[5,6]$. However, there are still about $30 \%$ of newly diagnosed patients with metastasis [7,8]. Besides,
ccRCC is insensitive to traditional radiotherapy and chemotherapy. Molecular targeting therapy such as VEGF-tyrosine kinase inhibitors[9] and mammalian target of rapamycin (mTOR) inhibitors $[10,11]$ is used to treat advanced ccRCC and metastatic ccRCC patients. Unfortunately, a part of patients has not shown satisfactory improvement due to tumor recurrence and drug resistance.

Recently, immunotherapy, as a newly developed approach, has changed the treatment pattern of advanced ccRCC [12-14]. Immunotherapy mainly focused on inhibiting tumor immune escape. Tumor cells can avoid being recognized and attacked by the 
immune system through various mechanisms including recognition of tumor specific antibodies as autoantigens, low immunogenicity of tumor cells, and tumor-induced immunosuppression, which is a vital strategy for tumor growth and progression $[15,16]$. At present, most studies have paid attention to relieve tumor-induced immunosuppression. Tumor-induced immunosuppression is mainly regulated through two mechanisms. The first mechanism is that immunosuppressive cells accumulate around the tumor and secrete immunosuppressive factors to inhibit immune response [17-19]. Another mechanism is that the tumor inhibits the activation of effector $\mathrm{T}$ lymphocytes via promoting the expression of immunosuppressive molecules or their receptors, such as PD-1/PD-L1 and CTLA4. PD-1/PD-L1 inhibitor is one of the most important medicines of immunotherapy. Some studies showed that PD-1/PD-L1 inhibitor could obviously benefit a subset of patients and prolong survival time in various tumors $[20,21]$. However, it has been reported that the therapeutic effect and effectiveness of PD-1/PD-L1 antagonist was low in some tumors due to the influence and function of the tumor microenvironment (TME) [22,23]. TME is composed of the vasculature, extracellular matrix (ECM), tumor cells, and a huge number of non-malignant cells $[24,25]$. In addition, various signal molecules form a complex signal network to maintain the internal connections in the TME [26,27]. Tumor-infiltrating immune cells (TIICs) are the most important part of non-malignant in the TME, which mainly include dendritic cells (DCs), natural killer (NK) cells, T cells, B cells, monocytes, macrophages, mast cells, neutrophil, and so on. Previous studies proved that TIICs played a vital role in the occurrence and development of tumors. Hu et al reveal that CD39(+)yסTregs could suppress anti-tumor immune via an adenosine-mediated pathway in colorectal cancer [28]. It has been reported that neutrophils inhibited tumor growth and progression via suppressing tumor-associated inflammatory responses [29]. In ccRCC, a previous report demonstrated that infiltrating CD4+ T cells promoted tumor cell proliferation via up-regulating TGF- $\beta 1$ expression [30]. However, the functions and molecular mechanisms of TIICs are still unclear due to the diversity of TIICs and complicacy of TME in ccRCC.

In this study, CIBERSORT, an online analytical tool for TIICs, was utilized to assess the relative abundance of 22 types of TIICs using mRNA expression data from the TCGA KIRC dataset. Then, the Cox proportional hazard regression model was constructed to divide ccRCC patients into high-risk group and low-risk group according to clinical data and abundance of TIICs. We then further screened differentially expressed genes (DEGs) between high-risk group and low-risk group and identified hub genes. Our study attempted to find key genes significantly associated with TIICs and tumor progression, which might become the potential biomarkers and therapeutic targets for immunotherapy in ccRCC.

\section{Material and methods}

\section{Data collection and processing}

All sequencing data and clinicopathological data were obtained from The Cancer Genome Atlas (TCGA) database (https://www.cancer.gov/tcga) and normalized via the $\mathrm{R}$ program. Moreover, the details of the data processing were described as a flow diagram (Figure 1).

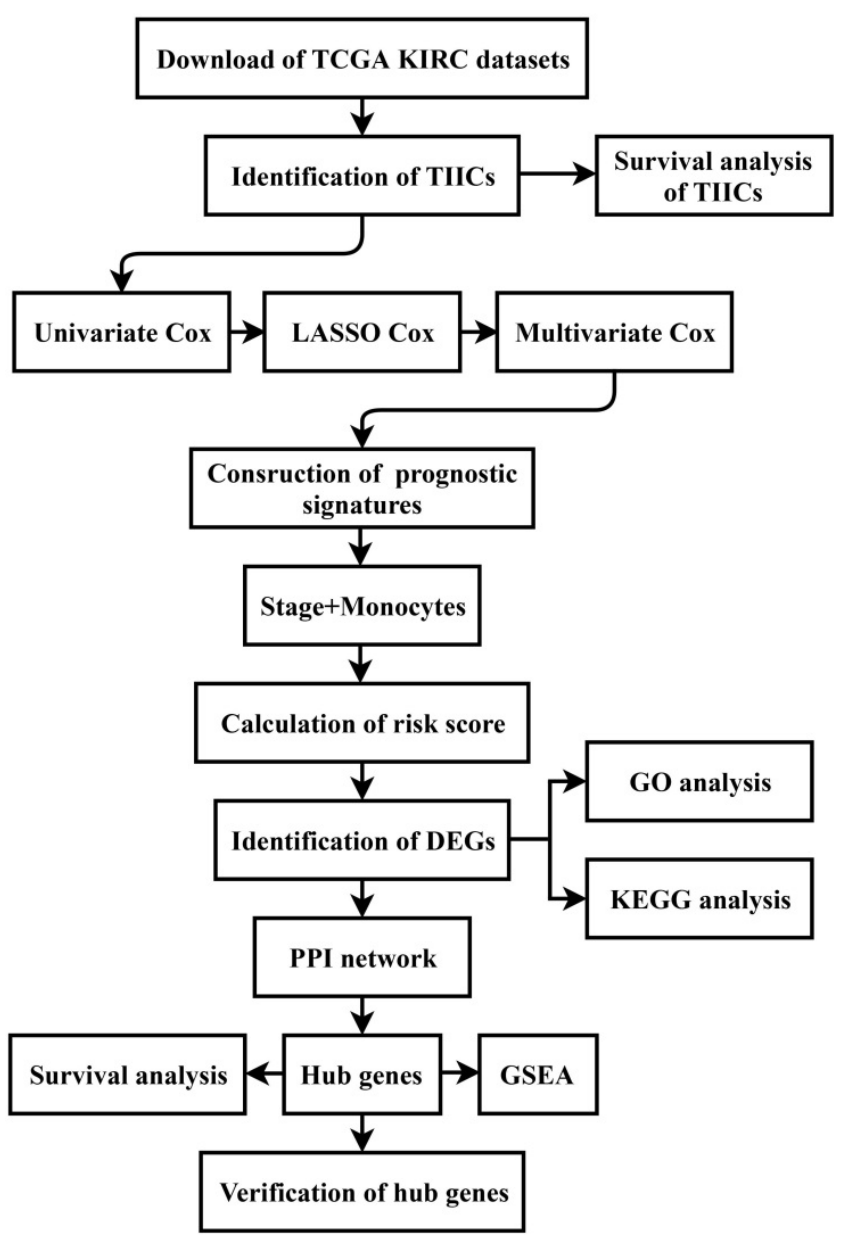

Figure 1. Flow diagram of this study. The details of data collection and analysis were exhibited in a flow diagram. TCGA: The Cancer Genome Atlas; KIRC: Kidney Renal Clear Cell Carcinoma; TIICs: tumor-infiltrating immune cells; LASSO: least absolute shrinkage and selection operator; DEGs: differentially expressed genes; GO: Gene Ontology; KEGG: Kyoto Encyclopedia of Genes and Genomes; PPI: protein-protein interaction; GSEA: gene set enrichment analysis. 
Assessment of tumor-infiltrating immune cells (TIICs)

CIBERSORT algorithm was used to infer the relative abundance of 22 TIICs in each ccRCC sample according to the normalized gene expression data. The gene expression data were submitted to the CIBERSORT web portal (http://cibersort.stanford. edu), with the algorithm run using the LM22 signature matrix at 1,000 permutations. P-value $<0.05$ was considered statistically significant.

\section{Survival analysis of TIICs and hub genes in ccRCC}

The survival data were obtained from the TCGA KIRC dataset. All ccRCC samples were divided into high group and low group according to the median abundance of TIICs or the median expression of hub genes. GraphPad Prism (version 7.0) was used to draw the survival curves of TIICs. GEPIA (http://gepia.cancer-pku.cn), an online tool, was used to perform survival analysis of hub genes. P-value $<0.05$ was considered statistically significant.

\section{Construction of Cox proportional hazard regression model}

The TCGA KIRC cohort was randomly divided into two groups (training set and test set). In the training set, univariate cox regression analysis was performed to identify TIICs, which were associated with overall survival (OS) via the "survival" package (https://CRAN.R-project.org/package=survival) in $R$. Then, based on the results of univariate Cox regression analysis, least absolute shrinkage and selection operator (LASSO) cox regression with a 10fold cross validation was used to further screen the important factors via "glmnet" [31,32] package. Next, we conducted multivariate cox regression to calculate the coefficient and construct a prognostic signature. Risk scores of ccRCC patients were calculated via the "glmnet" package based on the coefficient of each factor. We divided ccRCC patients into high-risk group and low-risk group according to the median of risk score and analyzed the prognostic role of the prognostic signature in both training set and test set.

\section{Identification and functional annotation of differentially expressed genes (DEGs) between high-risk cluster and low-risk cluster}

The "DESeq2" [33] package was used to identify the DEGs between high-risk group and low-risk group. $|\log \mathrm{FC}|>1.0$ and P-value $<0.05$ were selected as cutoff criterion. Then, the "clusterProfiler" [34] package was used to perform Gene Ontology (GO) and Kyoto Encyclopedia of Genes and Genomes
(KEGG) enrichment analysis in R. P-value $<0.05$ was considered statistically significant.

\section{Construction of protein-protein (PPI) network}

All DEGs were submitted to the STRING online tool (https://string-db.org) and construct a PPI network to search hub genes. Then, we utilized cytoscape software [35] to perform network analysis and calculate the degree of each DEG. Molecular Complex Detection (MCODE) plug [36] was used to screen important key modules. Genes with degree > 10 were identified as hub genes.

\section{Gene set enrichment analysis (GSEA)}

The ccRCC samples were divided into two groups (high expression group and low expression group) based on the median expression of hub genes. The gene expression matrix was input to GSEA software [37, 38] for further enrichment analysis. Nominal $\mathrm{P}<0.05$ and false discovery rate (FDR) $<$ $25 \%$ were selected as cutoff criteria.

\section{Human ccRCC samples}

A total of 24 ccRCC samples were obtained from Wuhan Union Hospital between 2017 and 2018. All samples were stored at $-80{ }^{\circ} \mathrm{C}$ until use. All patients signed a written consent form and the study was approved by the ethics committee of Union Hospital and Huazhong University of Science and Technology.

\section{RNA extraction and $\mathrm{qRT}$-PCR}

Total RNA was extracted from ccRCC tissue samples using Trizol Reagent (Sigma, USA). The NanoDrop Lite UV-Vis Spectrophotometer (Thermo Scientific, USA) was used to detect the concentration and purity of total RNA. Then, cDNA was synthesized using qPCR RT Kit (Vazyme, China). After that, quantitative realtime PCR (qRT-PCR) was performed to amplify cDNA with specific primers and the data were normalized to GAPDH. Primer sequences were listed as following:

GAPDH Forward: 5'-GCACCGTCAAGGCTG AGAAC-3'; GAPDH Reverse: 5'-TGGTGAAGACGC CAGTGGA-3'; MMP9 Forward: 5'-AGACCTGGG CAGATTCCAAAC-3'; MMP9 Reverse: 5'-CGGCAA GTCTTCCGAGTAGT-3'; F2 Forward: 5'-CACGGC TACGGATGTGTTCTG-3'; F2 Reverse: 5' ${ }^{\prime}$-ACCC TCAGCACAGTTACCTTC-3'; HP Forward: 5'-CAG CACAGTCCCCGAAAAGAA-3'; HP Reverse: 5' CAGTCGCATACCAGGTGTCC-3'; CXCL13

Forward: 5'-GCTTGAGGTGTAGATGTGTCC-3'; CXCL13 Reverse: 5'-CCCACGGGGCAAGATTTG AA-3'; IGFBP1 Forward: 5'-TTGGGACGCCATCAG TACCTA-3'; IGFBP1 Reverse: 5'-TTGGCTAAA CTCTCTACGACTCT-3'; VTN Forward: 5'-CGGGGA TGTGTTCACTATGCC-3'; VTN Reverse: 5'-GTGTCT 
GCTCAGGATTCCCTT-3'; VGF Forward: 5'-GGA ACTGCGAGATTTCAGTCC-3'; VGF Reverse: 5'-GTG CGGGTTTCCGTCTCTG-3'; MFI2 Forward: 5'-ACC TCCTATTACGCCGTGG-3'; MFI2 Reverse: 5'-AGG GACTCAGAGTAACTGGTC-3';SAA1 Forward: 5'CATGCTCGGGGGAACTAT-3'; SAA1 5'-TACCCATTGTGTACCCTCTCC-3'.

\section{Western blotting (WB)}

In brief, the proteins of tissues were extracted with the radio-immunoprecipitation assay (RIPA) lysis buffer (Beyotime, China) containing $1 \mathrm{mM}$ Phenylmethylsulfonyl fluoride (PMSF, Beyotime, China). The concentration of protein was detected using the BCA assay kit (MCE, China). 50ug of protein was subjected to $10 \%$ SDS-PAGE and transferred to PVDF membranes (Millipore, USA). Then, the membranes were blocked in 5\% nonfat dried skimmed milk for $1.5 \mathrm{~h}$ at room temperature. After that, the PVDF membranes were incubated with primary antibodies containing VGF (1:1000, Abcam, England, ab74140) and GAPDH (1:50000, Abclonal, China, AC002) overnight at $4{ }^{\circ} \mathrm{C}$. Finally, the membranes were incubated with corresponding secondary antibodies (1:3000, Proteintech, China, SA00001-1 and SA00001-2) for 1.5h at room temperature and visualized with the ChemiDoc-XRS+ system (Bio-Rad, USA).

\section{Immunohistochemistry (IHC) assay}

IHC was performed as previously described [39]. Firstly, ccRCC samples were fixed in formalin, dehydrated, and embedded in paraffin. Secondly, sample sections were incubated with corresponding primary antibodies overnight at $4{ }^{\circ} \mathrm{C}$. Thirdly, sample sections were washed with PBS three times. Finally, sample sections were incubated with corresponding secondary antibodies at room temperature for $2 \mathrm{~h}$. Moreover, ImageJ software was used to calculate the mean OD value of cells with positive staining.

The primary antibodies and secondary antibodies were as following: MMP9 (1:100, Abclonal, China, A0289); IGFBP1 (1:150, Abclonal, China, A11109); CD14 (1:200, Proteintech, China, 60253-1-lg); secondary antibodies (1:3000, Proteintech, China, SA00001-1 and SA00001-2).

\section{Statistical analysis}

All data were presented as mean \pm SD with three independent experiments. The student's $t$ test was used for two group data. Analysis of variance (ANOVA) was used to analyze multiple groups of data and least - significant difference (LSD) was used to analyze the difference between groups. All statistical analyses were performed using GraphPad Prism software (GraphPad Software, Inc., La Jolla,
CA, USA). $\mathrm{P}<0.05$ was considered to be statistically significant.

\section{Results}

\section{Distribution of TIICs in CCRCC and prognostic role of TIICs}

CIBERSORT was performed to identify the landscape of 22 TIICs in ccRCC. As shown in Figure 2A, tumors contained abundant fractions of CD8+ T cells $(21.5 \%)$, M2 macrophages $(20.3 \%)$, resting memory CD4+ T cells (16.1\%), M1 macrophages $(8.4 \%)$ and monocytes $(4.9 \%)$, whereas the fractions of naive CD4+ $\mathrm{T}$ cells $(0.00 \%)$, memory B cells $(0.02 \%)$, eosinophils $(0.05 \%)$ and activated dendritic cells $(0.13 \%)$ were low. The correlation heat map showed that the proportions of different TIICs subtypes were not strongly correlated (Figure 2B). Moreover, survival analysis displayed that high proportion of monocytes $(p=0.028, H R=0.68)$, resting mast cells (resting MCs) $(\mathrm{p}=0.004, \mathrm{HR}=0.61)$ and resting dendritic cells (resting DCs) $(p=0.013, \mathrm{HR}=0.65)$ predicted better OS (Figure 2C, E, F). However, patients with high proportion of follicular helper $\mathrm{T}$ (Tfh) cells $(\mathrm{p}=0.016, \mathrm{HR}=1.51)$ or regulatory $\mathrm{T}$ cells (Tregs) $(p=0.042, H R=1.42)$ had shorter OS time (Figure 2D, G).

\section{Construction of Cox proportional hazard regression model}

Firstly, we used univariate cox proportional hazard regression model to preliminarily screen important clinicopathological characteristics and TIICs which are related to OS in training set. Univariate analysis showed that age $(p=0.079$, $\mathrm{HR}=1.6, \mathrm{CI}$ : $0.95-2.70)$, grade $(\mathrm{p}=0.001, \mathrm{HR}=1.79, \mathrm{CI}$ : 1.27-2.52), TNM stage $(\mathrm{p}=0.000, \quad \mathrm{HR}=1.83, \quad \mathrm{CI}$ : $1.47-2.27)$, T stage ( $\mathrm{p}=0.000, \mathrm{HR}=1.82, \mathrm{CI}: 1.40-2.36), \mathrm{N}$ stage $(p=0.000, H R=5.19, C I$ : 2.46-10.98), M stage $(\mathrm{p}=0.000, \quad \mathrm{HR}=4.21, \mathrm{CI}:$ 2.53-7.00), plasma cells $(\mathrm{p}=0.074, \mathrm{HR}=1.55, \mathrm{CI}: 0.96-2.52)$, regulatory $\mathrm{T}$ cells $(p=0.025, H R=1.76, C I: 1.07-2.88)$, monocytes $(p=0.000$, $\mathrm{HR}=0.39$, CI: $0.23-0.64)$, resting dendritic cells $(\mathrm{p}=0.047, \mathrm{HR}=0.61, \mathrm{CI}: 0.37-0.99)$ and resting mast cells $(p=0.063, \quad H R=0.63, \quad C I: 0.39-1.03)$ were significantly related to prognosis (Table 1). Then, based on the LASSO cox regression model, four indexes (TNM stage, $\mathrm{N}$ stage, $\mathrm{M}$ stage and monocytes) with nonzero coefficient were selected as candidate prognostic indexes (Figure 3A, B). Finally, as shown in Figure 3C, TNM stage (coefficient $=0.58, H R=1.79$, CI: $\quad 1.43-2.25, \quad \mathrm{p}<0.001)$ and monocytes (coefficient $=-0.96, \mathrm{HR}=0.38, \mathrm{CI}: 0.23-0.65, \mathrm{p}<0.001$ ) were selected to construct risk index and prognostic signature via multivariate cox regression analysis. The 
risk index $=0.58^{*} \mathrm{TNM}$ stage $-0.96^{*}$ Monocytes. Then, we divided ccRCC patients into two clusters (high risk/TNM ${ }^{\text {high }}$ Monocytes ${ }^{\text {low }}$ cluster and low risk/TNM ${ }^{\text {low} M o n o c y t e s ~}{ }^{\text {high }}$ cluster) in both training set and test set according to the risk score. Survival analysis indicated that patients with high risk index had worse prognosis (Figure 3D, E) both in training set $(\mathrm{HR}=2.96, \mathrm{CI}: 1.82-4.83, \mathrm{p}<0.001)$ and test set ( $\mathrm{HR}=3.03, \mathrm{CI}: 1.88-4.88, \mathrm{p}<0.001)$.

\section{Identification of DEGs between high risk/TNM ${ }^{\text {high Monocytes }}{ }^{\text {low }}$ cluster and low risk/TNMlow/Monocytes ${ }^{\text {high }}$ cluster}

A total of 216 genes were identified as DEGs with $|\log \mathrm{FC}|>1.0$ and P-value $<0.05$. There were 204 up-regulated genes and 12 down-regulated genes in the high-risk cluster (Figure 4A).
Table 1. Univariate cox regression analysis of TIICs

\begin{tabular}{lllll}
\hline Variate & HR & $95 \%$ CI Low & $95 \%$ CI High & P-value \\
\hline Age & 1.60 & 0.95 & 2.70 & 0.079 \\
Gender & 0.85 & 0.52 & 1.39 & 0.517 \\
Grade & 1.79 & 1.27 & 2.52 & 0.001 \\
Stage & 1.83 & 1.47 & 2.27 & 0.000 \\
$\mathrm{~T}$ & 1.82 & 1.40 & 2.36 & 0.000 \\
$\mathrm{M}$ & 4.21 & 2.53 & 7.00 & 0.000 \\
$\mathrm{~N}$ & 5.19 & 2.46 & 10.98 & 0.000 \\
B cells naive & 0.99 & 0.61 & 1.59 & 0.951 \\
Plasma cells & 1.55 & 0.96 & 2.52 & 0.074 \\
T cells CD8 & 0.75 & 0.46 & 1.21 & 0.234 \\
T cells CD4 memory resting & 0.87 & 0.54 & 1.40 & 0.556 \\
T cells follicular helper & 1.19 & 0.74 & 1.94 & 0.473 \\
T cells regulatory (Tregs) & 1.76 & 1.07 & 2.88 & 0.025 \\
T cells gamma delta & 0.71 & 0.44 & 1.15 & 0.163 \\
NK cells activated & 1.03 & 0.64 & 1.65 & 0.916 \\
Monocytes & 0.39 & 0.23 & 0.64 & 0.000 \\
Macrophages M0 & 1.27 & 0.79 & 2.06 & 0.329 \\
Macrophages M1 & 0.73 & 0.45 & 1.18 & 0.201 \\
Macrophages M2 & 0.84 & 0.52 & 1.36 & 0.474 \\
Dendritic cells resting & 0.61 & 0.37 & 0.99 & 0.047 \\
Mast cells resting & 0.63 & 0.39 & 1.03 & 0.063 \\
\hline TIICs: tumor-infiltrating immune & & & &
\end{tabular}

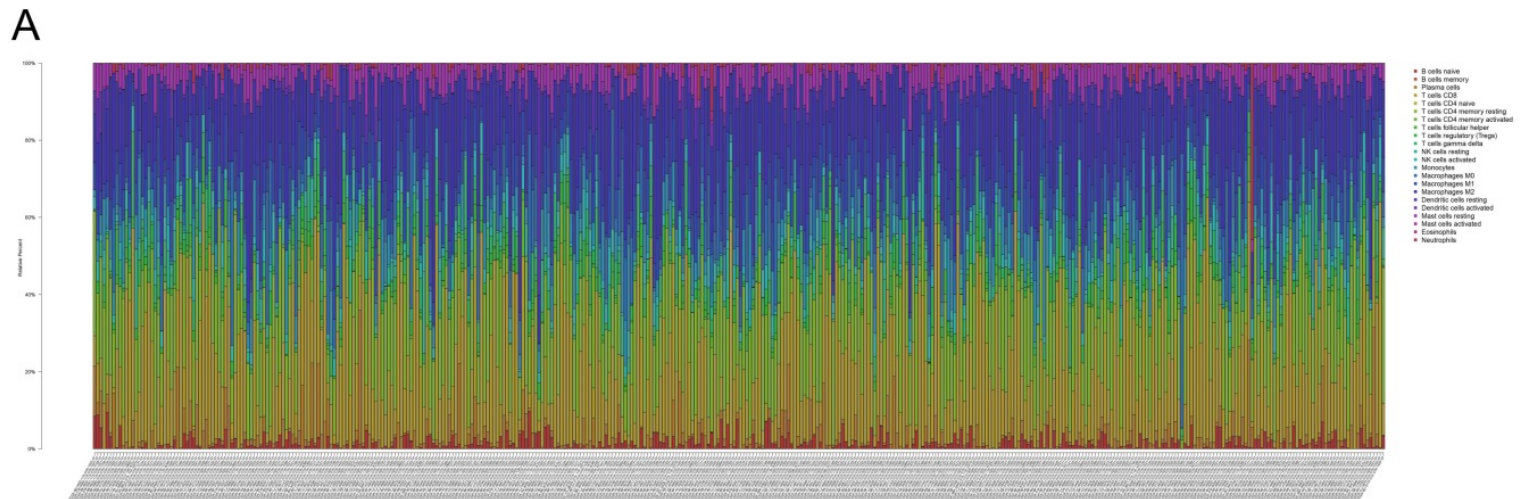

B
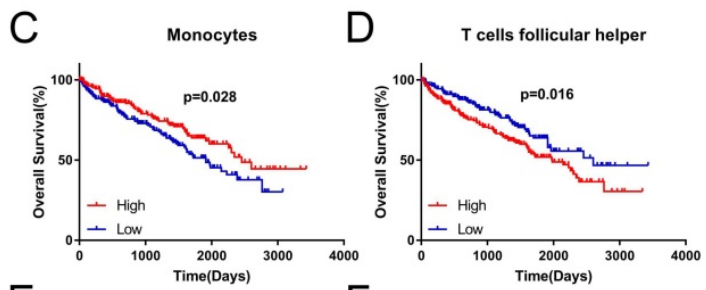

E
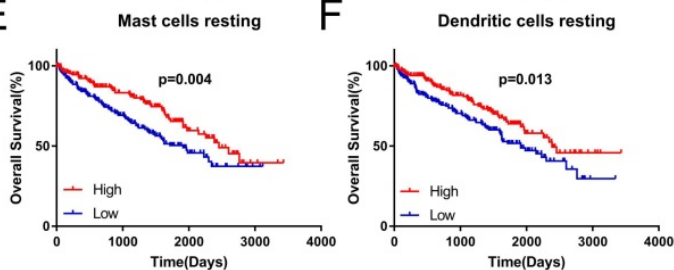

G

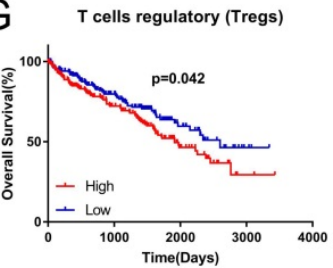

Figure 2. Distribution and prognostic role of TIICs in ccRCC. (A) Distribution of 22 TIICs in the TCGA KIRC dataset. (B) The heat map of correlation among 22 TIICs. (C-G) The prognostic value of TIICs. TIICs: tumor-infiltrating immune cells; ccRCC: clear cell renal cell carcinoma; TCGA: The Cancer Genome Atlas; KIRC: Kidney Renal Clear Cell Carcinoma. 
A

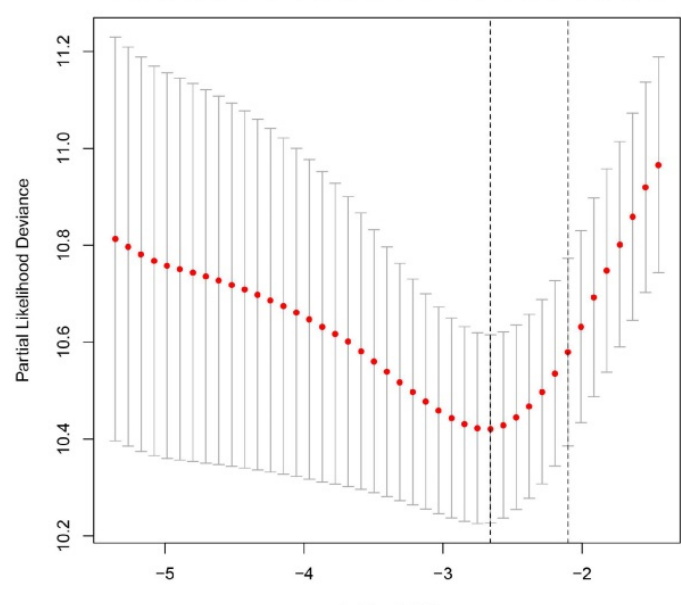

C

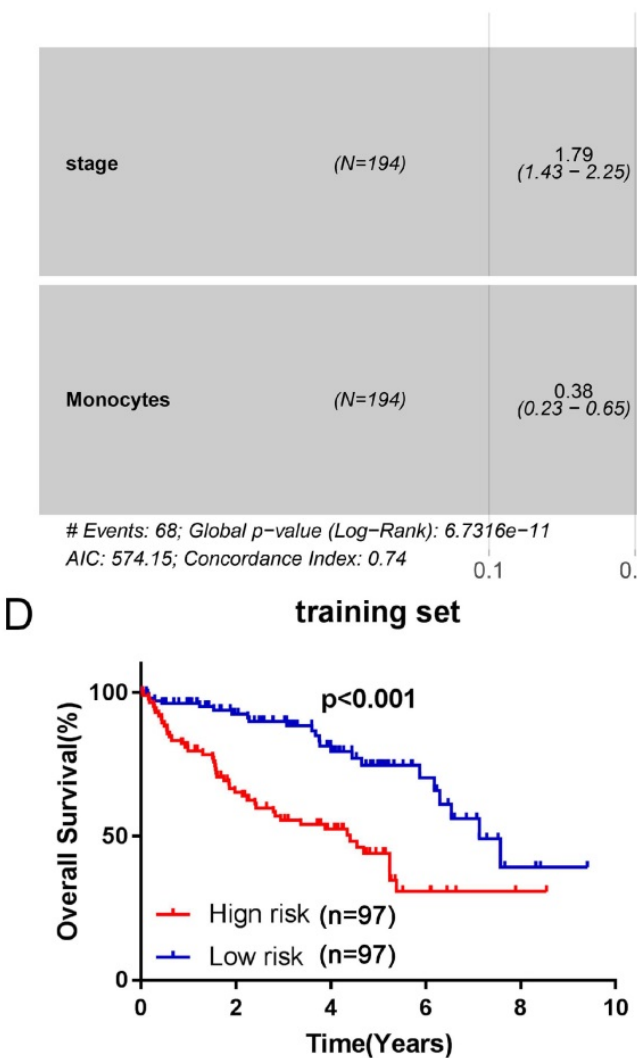

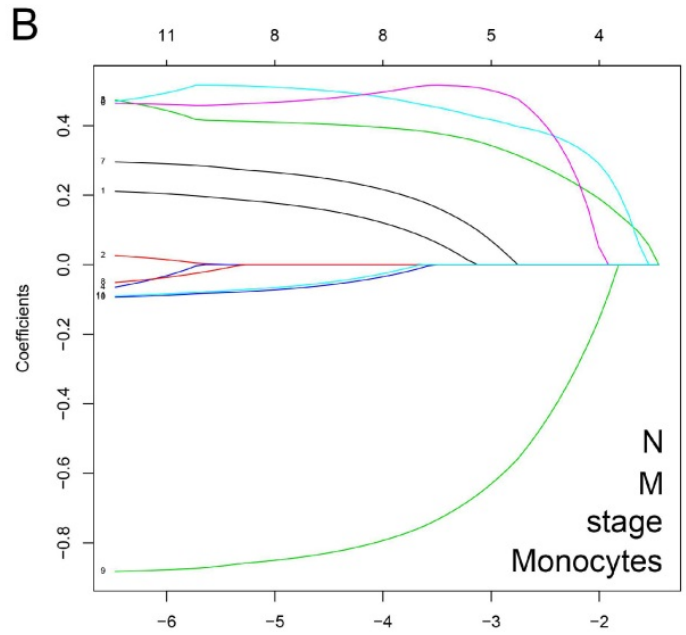

Hazard ratio

(azard ratio
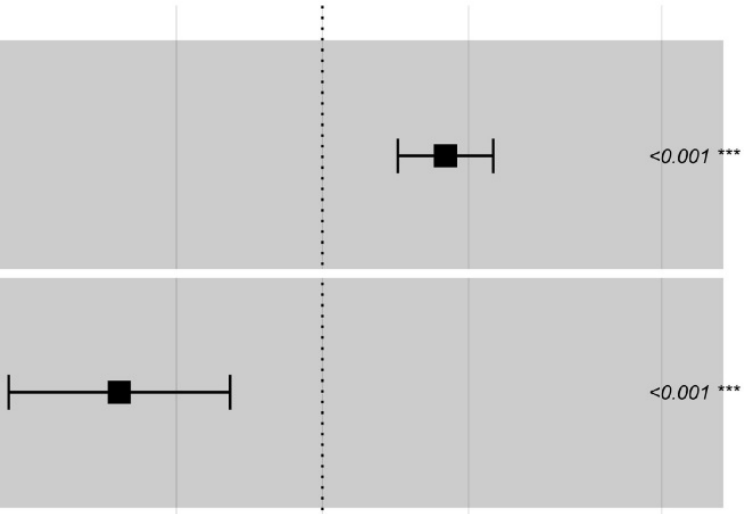

$E$

0.5

test set

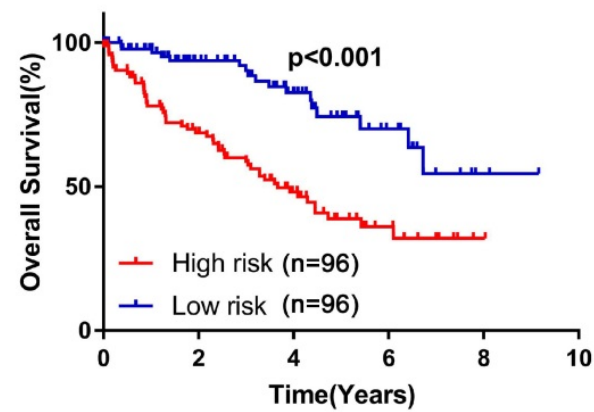

Figure 3. Construction of the Cox regression proportional hazard model. (A) Partial likelihood deviance for the LASSO coefficient profiles. (B) LASSO coefficient profiles of the clinical traits and TIICs. (C) Multivariate cox regression analysis of four variates (N, M, stage, and monocytes). Survival analysis was performed to verify the prognostic role of risk index for training set (D) and test set (E). LASSO: least absolute shrinkage and selection operator; TIICs: tumor-infiltrating immune cells.

\section{Function enrichment analysis of DEGs}

GO enrichment analysis indicated that the DEGs were mostly enriched in acute inflammatory response, humoral immune response, and regulation of immune effector process in the biological process (BP) group. Cellular component (CC) analysis showed that the DEGs were significantly enriched in blood microparticle, extracellular matrix, and endoplasmic reticulum lumen. Furthermore, molecular function (MF) results displayed that the DEGs were mainly related to receptor ligand activity, cytokine activity, and CCR chemokine receptor binding (Figure 4B). Moreover, KEGG pathway enrichment analysis also showed that the DEGs were obviously enriched in cytokine-cytokine receptor interaction, IL-17 signaling pathway, and TGF-beta signaling pathway. It was worth mentioning that the DEGs were enriched in salmonella infection and pathogenic Escherichia coli infection pathways (Figure 4C). 
A

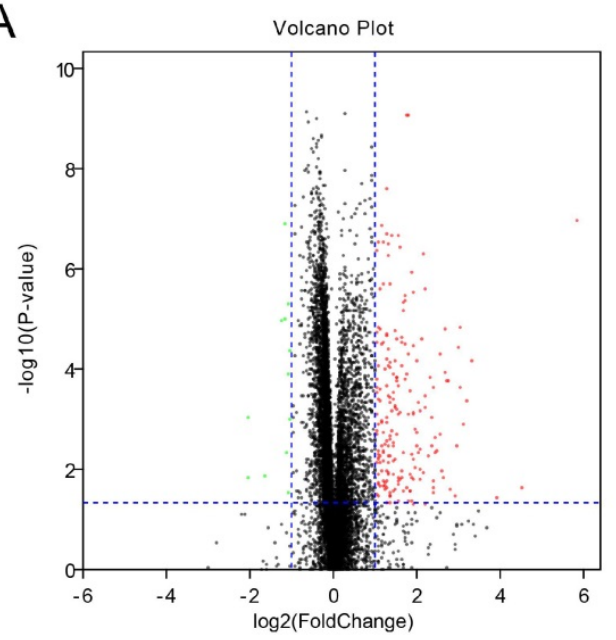

B

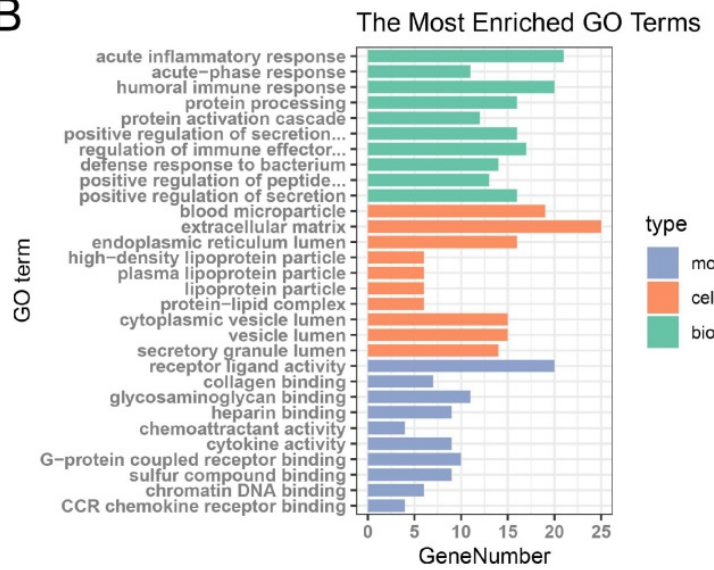

C

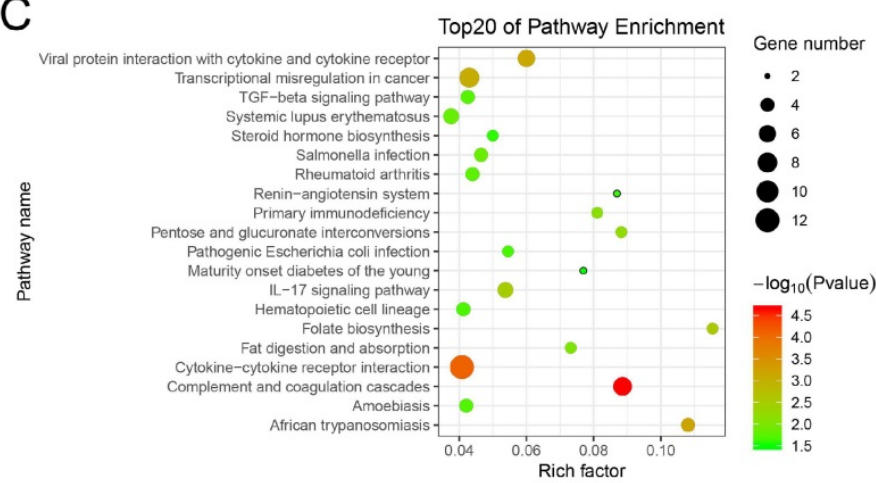

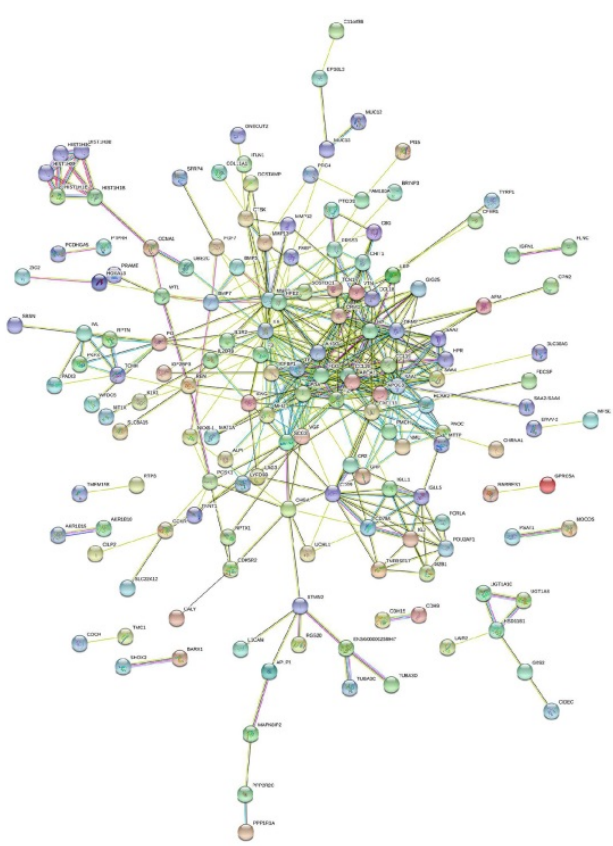

molecular function cellular component biological process

E

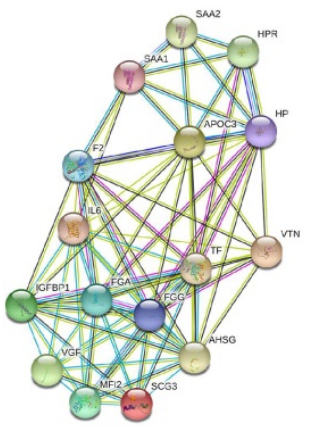

Figure 4. Functional annotation and PPI network analysis of DEGs. (A) Identification of DEGs between high-risk group and low-risk group. Red plots represent up-regulated genes, green plots represent down-regulated genes, and black plots represent unchanged genes. (B) GO annotation of DEGs. (C) KEGG pathway enrichment analysis of DEGs. (D) PPI network analysis of DEGs. (E) Identification of key modules via MCODE plug. PPI: protein-protein interaction; DEGs: differentially expressed genes; GO: Gene Ontology; KEGG: Kyoto Encyclopedia of Genes and Genomes; MCODE: Molecular Complex Detection.

\section{Construction of PPI network and identification of hub genes}

All DEGs were uploaded to STRING online database for constructing the PPI network. There were 157 nodes and 436 edges in the PPI network (Figure 4D). As shown in Figure 4E, the most important module contained 16 nodes (MFI2, SAA2, SCG3, AHSG, VTN, HP, IGFBP1, TF, IL6, FGG, HPR, FGA, VGF, F2, APOC3, and SAA1) and 76 edges. According to degree $>10$, a total of 24 genes were identified as hub genes (Table 2).

\section{Survival analysis of hub genes}

GEPIA was used to perform survival analysis (OS and DFS) of 24 hub genes. As shown in Figure 5 and Figure 6, high expression of MMP9, F2, HP, SAA1, CXCL13, IGFBP1, VTN, VGF, and MFI2 predicted poor OS and DFS. 
Table 2. PPI network of hub genes

\begin{tabular}{|c|c|c|c|c|c|c|c|c|}
\hline \multirow[t]{2}{*}{ Gene } & \multirow[t]{2}{*}{ Degree } & \multirow{2}{*}{$\begin{array}{l}\text { Betweenness } \\
\text { Centrality }\end{array}$} & \multirow{2}{*}{$\begin{array}{l}\text { Closeness } \\
\text { Centrality }\end{array}$} & \multirow{2}{*}{$\begin{array}{l}\text { Clustering } \\
\text { Coefficient }\end{array}$} & \multirow{2}{*}{$\begin{array}{l}\text { Neighborhood } \\
\text { Connectivity }\end{array}$} & \multirow[t]{2}{*}{ Stress } & \multicolumn{2}{|c|}{ Differential Analysis } \\
\hline & & & & & & & $\log \mathrm{FC}$ & P-value \\
\hline IL6 & 42 & 0.42 & 0.48 & 0.16 & 10.67 & 23056 & 1.08 & 0.000 \\
\hline MMP9 & 27 & 0.12 & 0.41 & 0.21 & 11.85 & 8260 & 1.79 & 0.000 \\
\hline $\mathrm{F} 2$ & 25 & 0.08 & 0.41 & 0.31 & 14.52 & 5774 & 1.97 & 0.000 \\
\hline $\mathrm{HP}$ & 24 & 0.03 & 0.38 & 0.43 & 15.38 & 3242 & 2.65 & 0.000 \\
\hline APOA1 & 23 & 0.03 & 0.40 & 0.48 & 15.26 & 3186 & 1.40 & 0.026 \\
\hline ORM1 & 22 & 0.04 & 0.36 & 0.39 & 13.73 & 4358 & 2.89 & 0.032 \\
\hline SAA1 & 22 & 0.06 & 0.40 & 0.35 & 14.45 & 4770 & 2.68 & 0.00 \\
\hline FGG & 20 & 0.02 & 0.39 & 0.53 & 16.00 & 2544 & 1.42 & 0.012 \\
\hline AHSG & 20 & 0.02 & 0.40 & 0.54 & 17.10 & 2894 & 2.37 & 0.023 \\
\hline APOC3 & 18 & 0.04 & 0.38 & 0.56 & 17.17 & 2526 & 2.48 & 0.004 \\
\hline FGA & 18 & 0.01 & 0.38 & 0.59 & 17.11 & 1978 & 1.87 & 0.005 \\
\hline $\mathrm{TF}$ & 17 & 0.01 & 0.39 & 0.61 & 17.41 & 1618 & 1.77 & 0.043 \\
\hline ORM2 & 15 & 0.00 & 0.33 & 0.63 & 16.33 & 364 & 1.65 & 0.026 \\
\hline CD19 & 14 & 0.08 & 0.37 & 0.32 & 10.00 & 5626 & 1.27 & 0.001 \\
\hline CXCL13 & 14 & 0.03 & 0.37 & 0.46 & 13.64 & 2362 & 1.78 & 0.000 \\
\hline IGFBP1 & 14 & 0.01 & 0.38 & 0.58 & 17.93 & 1150 & 1.42 & 0.000 \\
\hline CCL19 & 13 & 0.01 & 0.37 & 0.54 & 14.69 & 1600 & 1.34 & 0.000 \\
\hline CCL20 & 13 & 0.01 & 0.36 & 0.51 & 14.00 & 1264 & 1.02 & 0.030 \\
\hline VTN & 13 & 0.02 & 0.36 & 0.60 & 19.15 & 1582 & 2.40 & 0.017 \\
\hline SCG3 & 11 & 0.04 & 0.38 & 0.69 & 17.45 & 4654 & 1.90 & 0.015 \\
\hline VGF & 11 & 0.05 & 0.38 & 0.67 & 17.00 & 5026 & 1.93 & 0.001 \\
\hline IFNG & 11 & 0.03 & 0.37 & 0.38 & 15.18 & 2332 & 1.20 & 0.000 \\
\hline MFI2 & 11 & 0.02 & 0.36 & 0.67 & 16.45 & 876 & 1.85 & 0.003 \\
\hline SAA4 & 11 & 0.00 & 0.32 & 0.84 & 18.09 & 78 & 2.60 & 0.014 \\
\hline
\end{tabular}

PPI: protein - protein interaction; FC: fold change
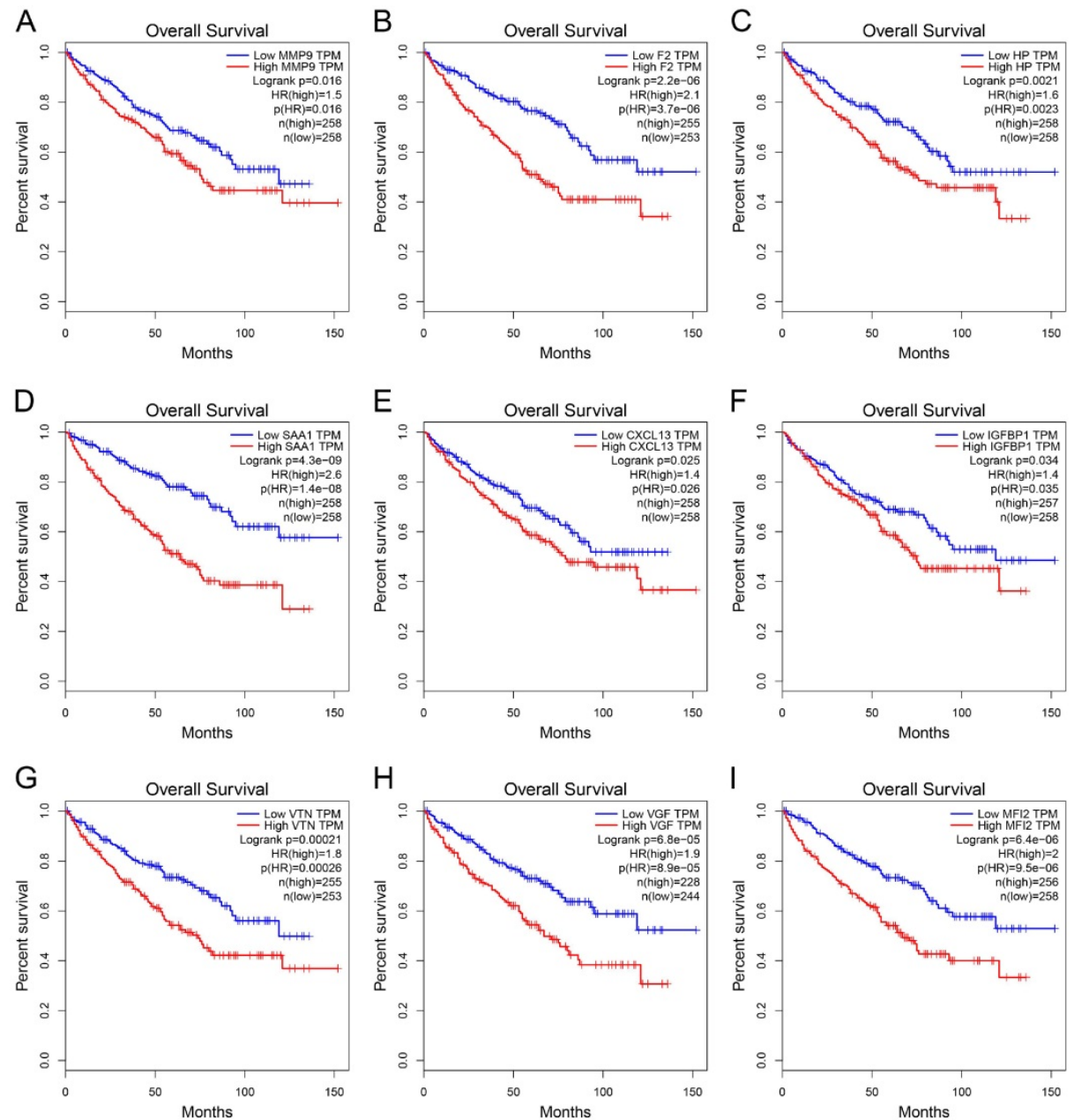

Figure 5. Survival analysis of hub genes for OS. (A) MMP9, Logrank $p=0.016, H R=1.5$. (B) $F 2$, Logrank $p=2.2 e-06, H R=2$.1. (C) $H P$, Logrank $p=0.0021$, $H R=1.6$. (D) $S A A 1$, Logrank $p=4.3 e-09, H R=2.6$. (E) $C X C L 13$, Logrank $p=0.025, H R=1.4$. (F) IGFBPI, Logrank $p=0.034$, HR=1.4. (G) VTN, Logrank $p=0.00021$, HR=1.8. (H) VGF, Logrank $p=6.8 \mathrm{e}-05$, $H R=1.9$. (I) $M F I 2$, Logrank $p=6.4 e-05, H R=2.0$. OS: overall survival; $H R$ : hazard ratio. 

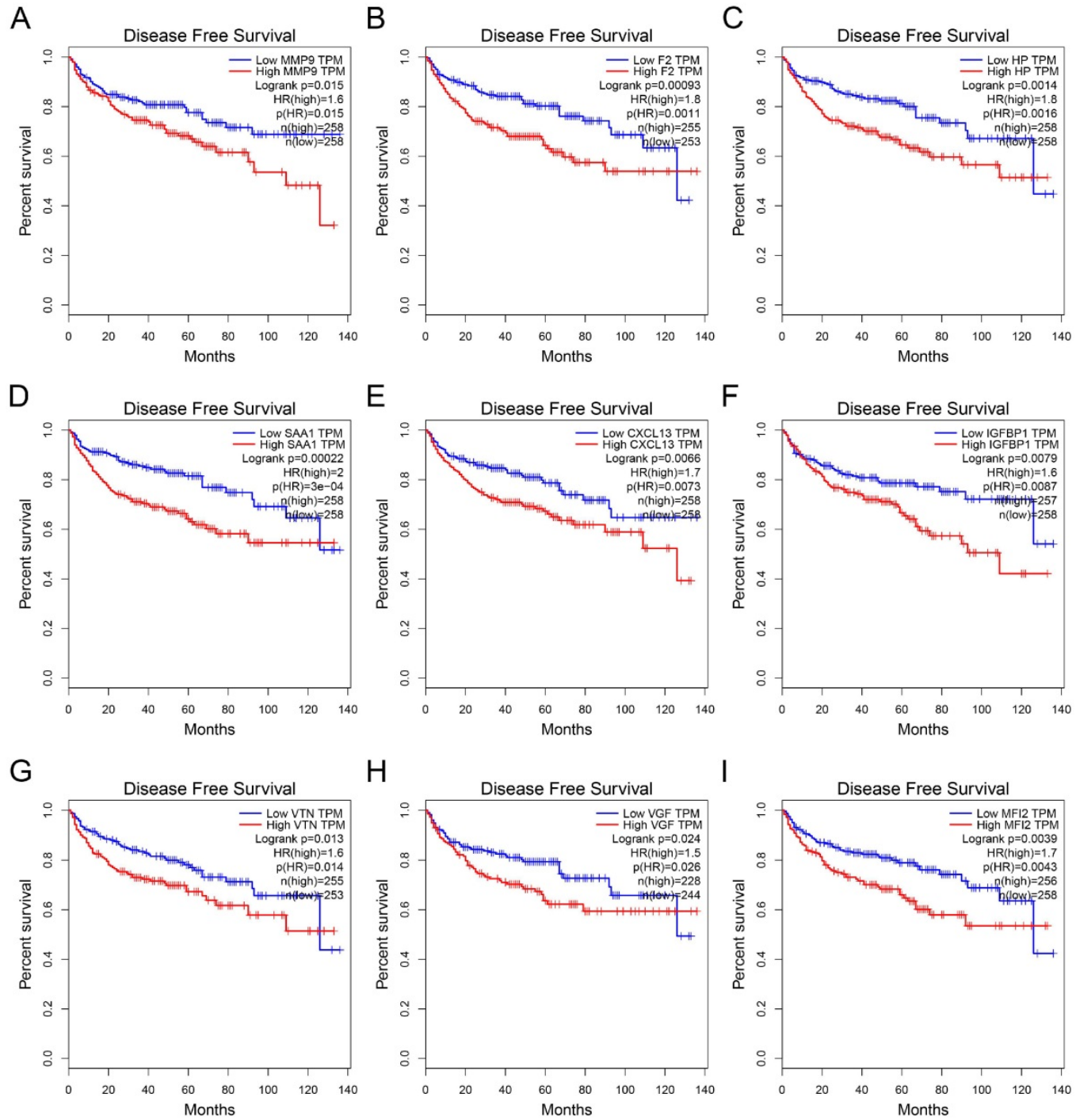

Figure 6. Survival analysis of hub genes for DFS. (A) MMP9, Logrank $p=0.015, H R=1.6$. (B) $F 2$, Logrank $p=0.00093, H R=1.8$. (C) $H P, L o g r a n k p=0.0014, H R=1.8$. (D) $S A A 1$, Logrank $p=0.00022$, HR=2.0. (E) $C X C L 13$, Logrank $p=0.0066$, HR=1.7. (F) IGFBP1, Logrank $p=0.0079, H R=1.6$. $(G) V T N$, Logrank $p=0.013$, HR=1.6. $(H) V G F$, Logrank $p=0.024$, $H R=1.5$. (I) $M F I 2$, Logrank $p=0.0039, H R=1.7$. DFS: disease-free survival; HR: hazard ratio.

\section{Validation of hub genes in ccRCC}

We utilized the IHC results of monocytes' signature (CD14) to identify the relative abundance of monocytes in ccRCC tissues (Figure 7A). Combined with the TNM stage, all ccRCC tissues were divided into high-risk group and low-risk group according to the risk index formula (Table 3). Then, qRT-PCR assay was used to validate the mRNA expression level of hub genes between high-risk group and low-risk group. As shown in Figure 7B-J, we found that the mRNA expressions of MMP9 and IGFBP1 were elevated in the high-risk group, and VGF was down-regulated in the high-risk group. However, differential analysis showed that VGF was up-regulated in the high-risk group, which was inconsistent with qRT-PCR results. Further WB assay showed that there was no significant difference between high risk group and low risk group (Figure S1). Therefore, we selected MMP9 and IGFBP1 for subsequent analysis. IHC results further showed that the protein expression level of MMP9 and IGFBP1 were up-regulated in the high-risk group (Figure 7K-L). Furthermore, the ccRCC samples were also divided into monocytes ${ }^{\text {high }}$ group and monocytes ${ }^{\text {low }}$ group according to the IHC results of CD14. We found that the expression level of MMP9 and IGFBP1 were elevated in the monocytes ${ }^{\text {low }}$ group (Figure 8A-C). Further correlation analysis showed that both MMP9 (Pearson $\mathrm{r}=-0.0 .43, \mathrm{P}=0.035$ ) and IGFBP1 (Pearson $\mathrm{r}=-0.39, \mathrm{P}=0.047$ ) were negatively correlated to CD14 (Figure 8D). These findings indicated that MMP9 and IGFBP1 were involved in tumor immune and progression. 

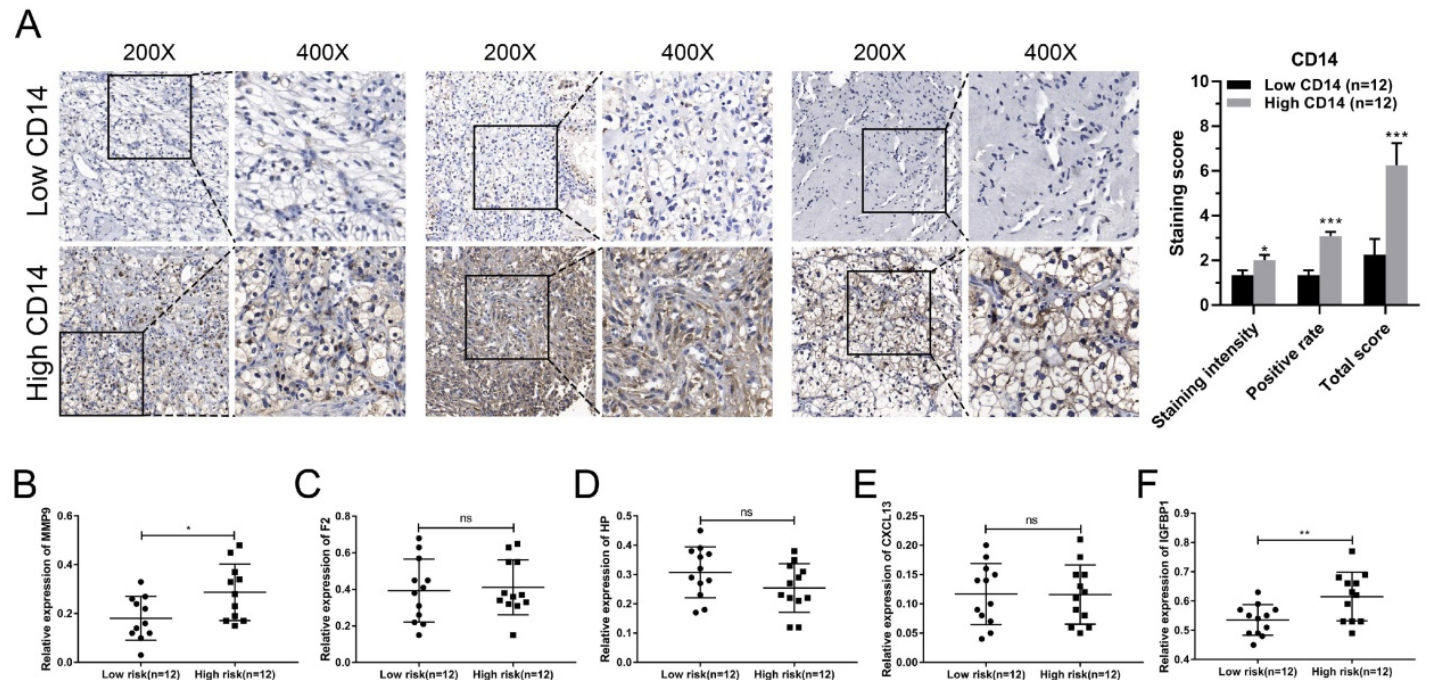

C
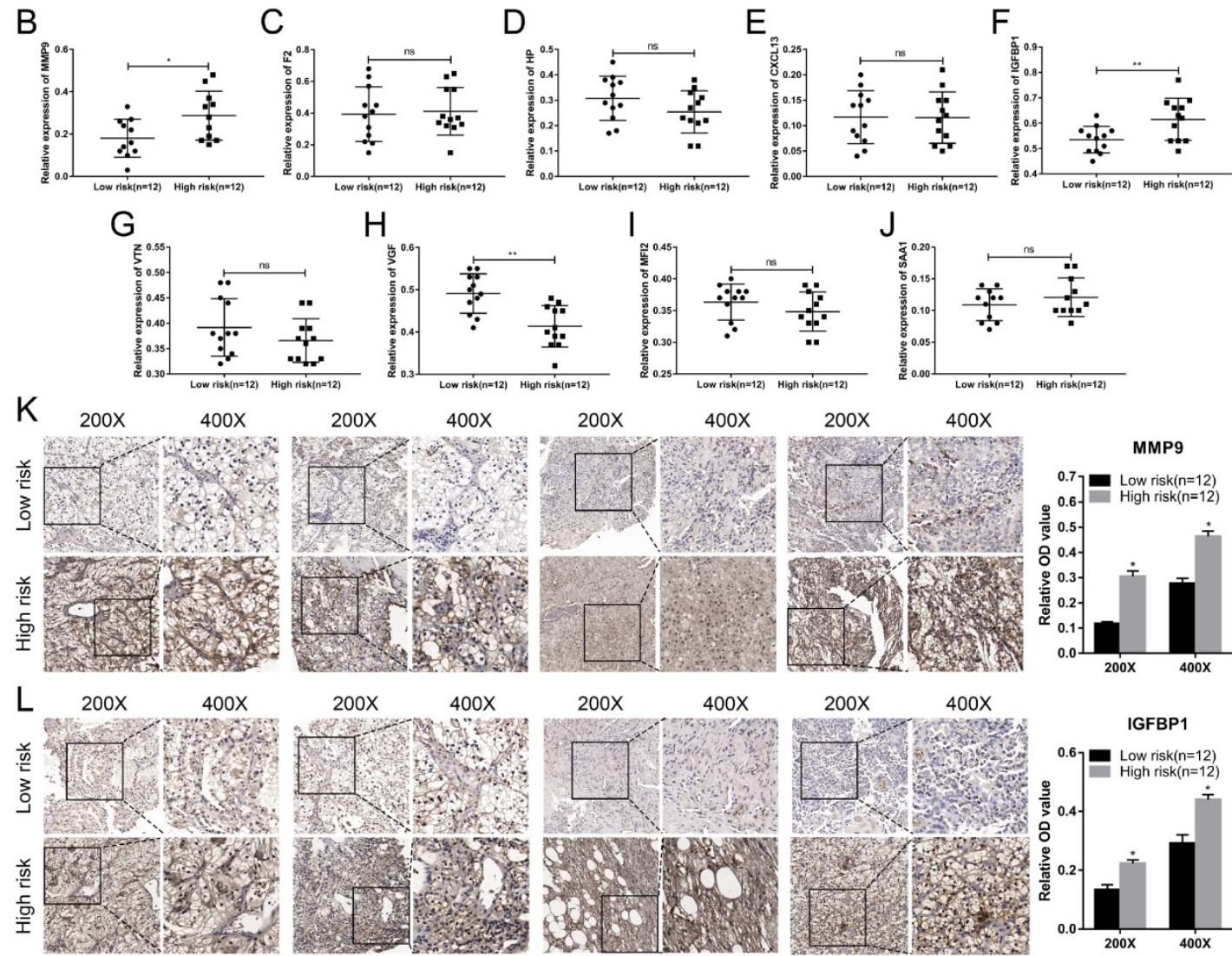

Figure 7. Validation of hub genes in the high-risk group and low-risk group. (A) ccRCC samples were divided into high monocytes group and low monocytes group based on the expression of CD14. Validation of the mRNA expression of MMP9 (B), F2 (C), HP (D), CXCL13 (E), IGFBP1 (F), VTN (G), VGF (H), MFI2 (I), and SAA1(J) between high-risk group and low-risk group. IHC assay was used to verify the protein expression of MMP9 (K) and IGFBPI (L) in ccRCC. ccRCC: clear cell renal cell carcinoma. ccRCC: clear cell renal cell carcinoma.

\section{GSEA of MMP9 and IGFBPI}

GSEA was performed to discover potential molecular mechanisms of MMP9 and IGFBP1 in tumor immune and progression. As shown in Figure 9A, MMP9 was mainly enriched in immune response regulating cell surface receptor signaling pathway, negative regulation of immune response pathway, and cytokine production involved in immune response pathway. In addition, IGFBP1 was significantly associated with negative regulation of immune effector process pathway and naive B cell vs monocyte pathway (Figure 9B).

\section{Discussion}

Nowadays, immunotherapy is used to treat advanced ccRCC or metastatic ccRCC, which prolong the overall survival time of patients. Many studies have shown that immunotherapy can activate the immune system and inhibit tumor progression [4042]. Unfortunately, there are still many patients whose state was not improved due to drug resistance and influence of tumor microenvironment (TME). At present, we recognize that tumor is not simply a cell mass composed of malignant cells but is actually composed of many non-malignant cells [43,44]. Tumor-infiltrating immune cell (TIIC) is one of the 
most important non-malignant cells, which involved in regulating tumor immune and immune escape. Several reports also revealed that TIICs were significantly correlated to the expression of immune checkpoints $[45,46]$. Therefore, further study on the role of TIICs may improve the efficacy of immunotherapy and find new therapeutic targets.

Table 3. The risk group of ccRCC samples

\begin{tabular}{llll}
\hline Patient ID & TNM stage & $\begin{array}{l}\text { Monocyte/ } \\
\text { dummy variable }\end{array}$ & $\begin{array}{l}\text { Risk score/ } \\
\text { Risk group }^{\text {A }}\end{array}$ \\
\hline Case-1 & 2 & $0.253 / 2$ & $-0.76 /$ Low \\
Case-2 & 3 & $0.201 / 2$ & $-0.18 /$ Low \\
Case-3 & 1 & $0.248 / 2$ & $-1.34 /$ Low \\
Case-4 & 2 & $0.210 / 2$ & $-0.76 /$ Low \\
Case-5 & 4 & $0.164 / 1$ & $1.36 /$ High \\
Case-6 & 2 & $0.189 / 1$ & $0.20 /$ High \\
Case-7 & 3 & $0.176 / 1$ & $0.78 /$ High \\
Case-8 & 4 & $0.168 / 1$ & $1.36 /$ High \\
Case-9 & 2 & $0.265 / 2$ & $-0.76 /$ Low \\
Case-10 & 1 & $0.241 / 2$ & $-1.34 /$ Low \\
Case-11 & 1 & $0.232 / 2$ & $-1.34 /$ Low \\
Case-12 & 2 & $0.201 / 2$ & $-0.76 /$ Low \\
Case-13 & 4 & $0.173 / 1$ & $1.36 /$ High \\
Case-14 & 2 & $0.198 / 1$ & $0.20 /$ High \\
Case-15 & 3 & $0.186 / 1$ & $0.78 /$ High \\
Case-16 & 3 & $0.157 / 1$ & $0.78 /$ High \\
Case-17 & 1 & $0.221 / 2$ & $-1.34 /$ Low \\
Case-18 & 2 & $0.158 / 1$ & $0.20 /$ High \\
Case-19 & 4 & $0.162 / 1$ & $1.36 /$ High \\
Case-20 & 2 & $0.206 / 2$ & $-0.76 /$ Low \\
Case-21 & 3 & $0.190 / 1$ & $0.78 /$ High \\
Case-22 & 1 & $0.259 / 2$ & $-1.34 /$ Low \\
Case-23 & 2 & $0.272 / 2$ & $1.36 /$ High \\
Case-24 & 4 & $0.191 / 1$ & Low \\
\hline
\end{tabular}

dummy variable ${ }^{\Psi}: 1$ represents the relative abundance of monocyte less than the median; 2 represents the relative abundance of monocyte more than the median. Risk group $^{\mathrm{A}}:$ High represents the risk score of patient less than the median; Low represents the risk score of patient more than the median.

In our study, the abundances of 22 TIICs were identified based on the gene sequencing data. Then, monocytes and the TNM stage were identified as independent prognostic factors and constructed the prognostic signature via cox regression analysis in cCRCC. Further studies uncovered that MMP9 and IGFBP1 were associated with tumor immune and progression.

Monocytes are mononuclear phagocytes that play an important role in the inflammatory response, immune response, and maintaining tissue homeostasis [47]. Recently, increasing studies indicated that tumor-infiltrating monocytes were significantly correlated to tumor progression and displayed diverse functions at different stages of tumor. Liu et al reported that CCL15 could recruit $\mathrm{CCR} 1^{+} \mathrm{CD} 14^{+}$monocytes to promote tumor immune escape and progression [48]. Similarly, Qian et al revealed that CCL2 facilitated tumor metastasis through recruiting Gr1-positive inflammatory monocytes in breast cancer [49]. It has been reported that tumor-infiltrating monocytes promoted invasion and migration of tumor cell via up-regulating S100A8 and S100A9 expression [50]. Monocytes also could differentiate into tumor-associated macrophages (TAM) to drive tumor growth and metastasis [51]. In addition, monocytes also acted as a tumor suppressor through recruiting NK cells [52,53], inhibiting Tregs function [54], and regulating cytotoxicity [55,56]. In the present study, we also found that monocytes served as an independent prognostic factor and ccRCC patients with high abundance of monocytes predicted better OS. These findings suggested that tumor-infiltrating monocytes may suppress ccRCC progression. Then, differential analysis and PPI network analysis indicated that MMP9 and IGFBP1 may act as mediators of monocytes in ccRCC.

MMP9 (matrix metallopeptidase 9), a member of matrix metallopeptidase superfamily, is mainly involved in local proteolysis of the extracellular matrix and leukocyte migration $[57,58]$. Numerous studies verified that MMP9 promoted tumor growth and metastasis in various cancers [59-61]. Furthermore, previous studies reported that MMP9 could regulate the biological functions of monocytes and their differential cells [62-65]. In this study, we also found that MMP9 was elevated in the TNM ${ }^{\text {high }}$ Monocytes ${ }^{\text {low }}$ group of ccRCC. Meanwhile, GSEA showed that high MMP9 group was significantly enriched in immune response pathway, cell surface receptor signaling pathway, and cytokine production pathway. These findings suggested that MMP9 may act as a regulator of cytokines and membrane receptors to mediate immune response in cCRCC.

IGFBP1 (insulin like growth factor binding protein 1) is a member of IGFBPs superfamily, which mainly regulates the bioavailability of IGF-1 and synthesizes in the liver and kidney $[66,67]$. It has been reported that IGFBP1 plays a dual role in both tumor growth and metastasis [68-70]. Moreover, previous studies revealed that IGFBP1 was involved in regulating cell migration [71]. Brandt et al uncovered that IGFBP1 and its fragments promoted human dermal fibroblasts migration [72]. Dorniak et al demonstrated that prostaglandins stimulated trophectoderm cell migration and attachment via regulating IGFBP1 expression [73]. Similarly, Irving et al also proved that IGFBP1 regulated the migration function of first trimester invasive trophoblasts by interacting with the RGD binding site of the $\alpha-5-\beta-1$ integrin [74]. These studies suggested that IGFBP1 was significantly associated with tumor progression and cell migration. In the present study, IGFBP1 was identified as a regulator for tumor progression and immune in ccRCC. Furthermore, GSEA uncovered 
that IGFBP1 was mainly involved in negative regulating of immune effector process pathway and naïve $\mathrm{B}$ cell vs monocyte pathway. These results indicated that IGFBP1 might regulate tumor progression and immune via mediating the biological functions of monocytes.
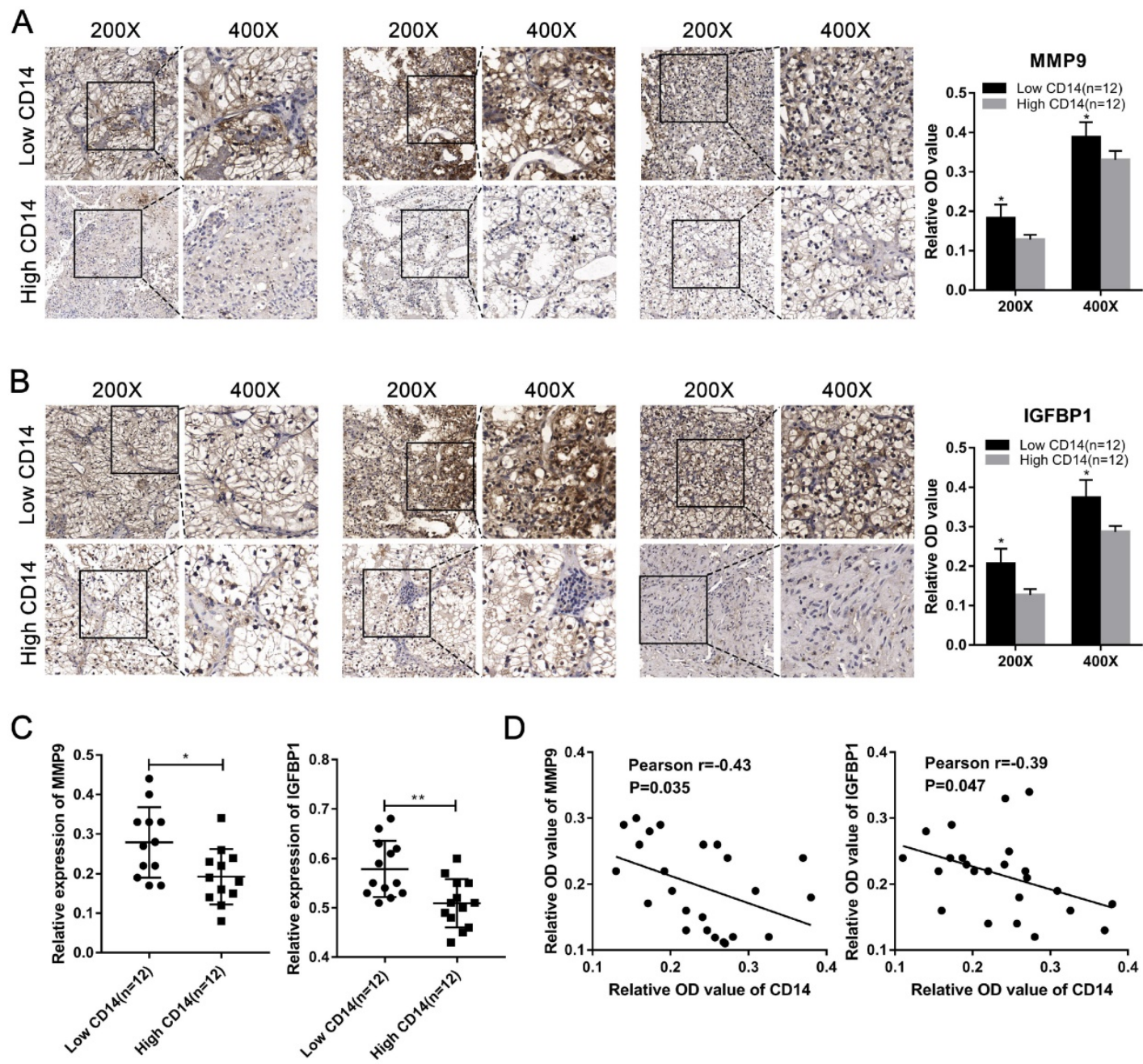

D
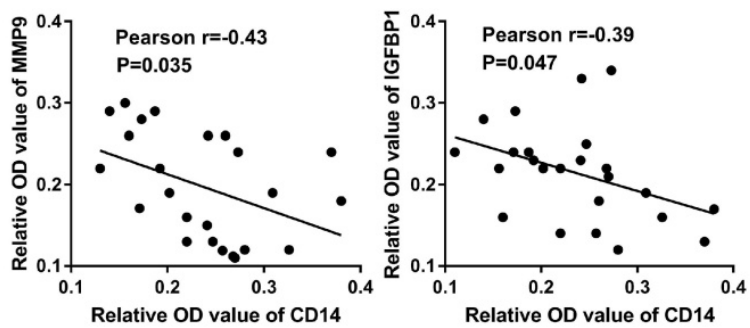

Figure 8. MMP9 and IGFBPI were up-regulated in the monocyteslow group and negative correlated to CD14. (A-C) Both protein expression and mRNA levels of MMP9/IGFBPI were elevated in the monocytes low group. (D) The protein expression levels of MMP9 (Pearson $r=-0.0 .43, P=0.035)$ and IGFBPI $(P e a r s o n ~ r=-0.39$, $P=0.047)$ were negative correlated to CD14.

$$
\text { A }
$$
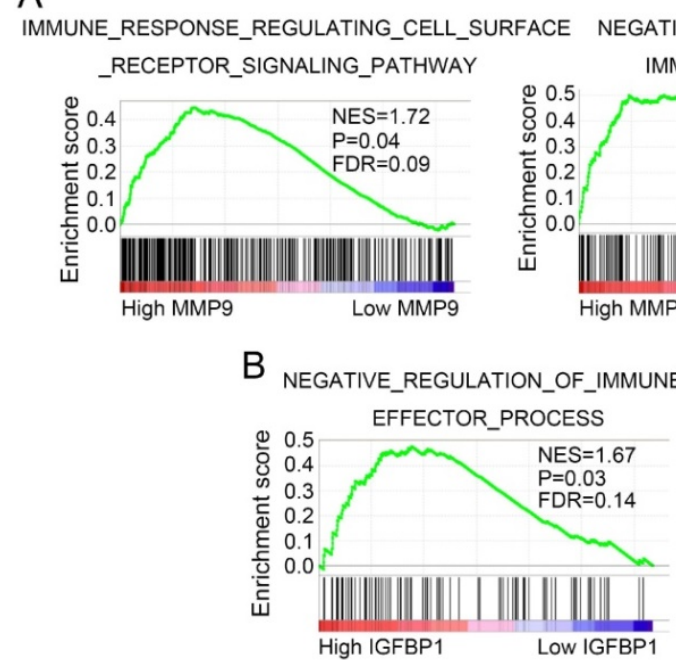
EGULATION_OF_

CYTOKINE_PRODUCTION_INVOLVED IN_IMMUNE_RESPONSE

B

NEGATIVE_REGULATION_OF_IMMUNE_

NAIVE_BCELL_VS_MONOCYTE_DN
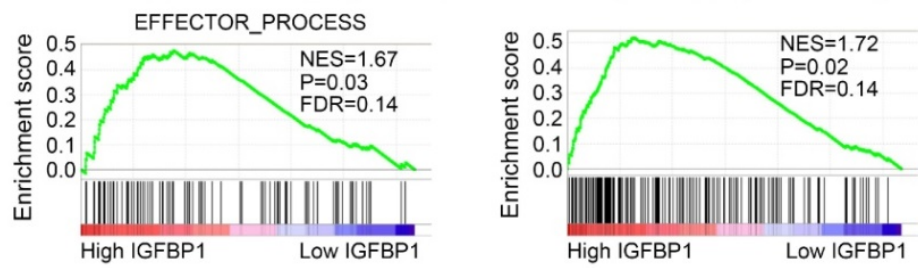

Figure 9. MMP9 and IGFBPI regulate immune related pathways. GSEA was performed to discover the potential mechanisms of MMP9 (A) and IGFBPI (B) in cCRCC. GSEA: gene set enrichment analysis; ccRCC: clear cell renal cell carcinoma. 
In conclusion, monocyte was identified as an independent protective factor in ccRCC. Further bioinformatics analysis revealed that MMP9 and IGFBP1 might act as regulators for monocytes migration and tumor immune. Moreover, GSEA indicated that MMP9 regulated immune response by mediating cell surface receptor and cytokine pathways and IGFBP1 was mainly involved in the regulation of monocytes. These findings suggested that MMP9 and IGFBP1 could become potential biomarkers and therapeutic targets for immunotherapy in ccRCC.

\section{Abbreviations}

ccRCC: clear cell renal cell carcinoma; TIICs: tumor-infiltrating immune cells; PPI: protein-protein interaction; RCC: renal cell carcinoma; mTOR: mammalian target of rapamycin; TME: tumor microenvironment; ECM: extracellular matrix; DCs: dendritic cells; NK: natural killer; TCGA KIRC: The Cancer Genome Atlas Kidney Renal Cell Carcinoma; DEGs: differentially expressed genes; OS: overall survival; LASSO: least absolute shrinkage and selection operator; GO: Gene Ontology; KEGG: Kyoto Encyclopedia of Genes and Genomes; MCODE: Molecular Complex Detection; GSEA: Gene set enrichment analysis; qRT-PCR: quantitative realtime PCR; IHC: Immunohistochemistry; ANOVA: Analysis of variance; LSD: least - significant difference; MCs: mast cells; Tfh: follicular helper T; Tregs: regulatory $\mathrm{T}$ cells; FC: fold change; BP: biological process; CC: cellular component; MF: molecular function; TAM: tumor-associated macrophages.

\section{Supplementary Material}

Supplementary figure S1.

http://www.jcancer.org/v12p2243s1.pdf

\section{Acknowledgements}

We would like to express our gratitude to all the members who participated in the discussion and assisted in this study. This study was supported by the Key Research and Development Plan in China (grant no. 2017YFB1303100), the National Natural Science Foundation of China (grant nos. 81672524, 81672528, and 81874090), the Hubei Provincial Natural Science Foundation of China (grant no. 2018CFA038), the Independent Innovation Foundation of Huazhong University of Science and Technology (grant no. 118530309) and the Clinical Research Physician Program of Tongji Medical College, Huazhong University of Science and Technology (grant no. 5001530015).

\section{Ethics Committee Approval and Patient Consent}

The authors are accountable for all aspects of the work in ensuring that questions related to the accuracy or integrity of any part of the work are appropriately investigated and resolved. The study was in accordance with the ethical standards formulated in the Helsinki Declaration and was approved by the ethics committee of Union Hospital and Huazhong University of Science and Technology. All patients enrolled completed the informed consent form.

\section{Competing Interests}

The authors have declared that no competing interest exists.

\section{References}

1. Rini BI, Campbell SC, Escudier B. Renal cell carcinoma. Lancet. 2009; 373: 1119-32.

2. Siegel RL, Miller KD, Jemal A. Cancer statistics, 2019. CA: a cancer journal for clinicians. 2019; 69: 7-34.

3. Campbell S, Uzzo RG, Allaf ME, Bass EB, Cadeddu JA, Chang A, et al. Renal Mass and Localized Renal Cancer: AUA Guideline. The Journal of urology. 2017; 198: 520-9.

4. Ljungberg B, Bensalah K, Canfield S, Dabestani S, Hofmann F, Hora M, et al. EAU guidelines on renal cell carcinoma: 2014 update. European urology. 2015; 67: 913-24.

5. Capitanio U, Montorsi F. Renal cancer. Lancet. 2016; 387: 894-906.

6. Van Poppel H, Da Pozzo L, Albrecht W, Matveev V, Bono A, Borkowski A, et al. A prospective, randomised EORTC intergroup phase 3 study comparing the oncologic outcome of elective nephron-sparing surgery and radical nephrectomy for low-stage renal cell carcinoma. European urology. 2011; 59: 543-52.

7. Crispen PL, Breau RH, Allmer C, Lohse CM, Cheville JC, Leibovich BC, et al. Lymph node dissection at the time of radical nephrectomy for high-risk clear cell renal cell carcinoma: indications and recommendations for surgical templates. European urology. 2011; 59: 18-23.

8. Gong J, Maia MC, Dizman N, Govindarajan A, Pal SK. Metastasis in renal cell carcinoma: Biology and implications for therapy. Asian journal of urology. 2016; 3: 286-92.

9. Hahn AW, Froerer C, VanAlstine S, Rathi N, Bailey EB, Stenehjem DD, et al. Targeting Bacteroides in Stool Microbiome and Response to Treatment With First-Line VEGF Tyrosine Kinase Inhibitors in Metastatic Renal-Cell Carcinoma. Clinical genitourinary cancer. 2018; 16: 365-8.

10. Turnbull JD, Cobert J, Jaffe T, Harrison MR, George DJ, Armstrong AJ. Activity of single-agent bevacizumab in patients with metastatic renal cell carcinoma previously treated with vascular endothelial growth factor tyrosine kinase inhibitors. Clinical genitourinary cancer. 2013; 11: 45-50.

11. Lalani AA, McGregor BA, Albiges L Choueiri TK, Motzer R, Powles T, et al. Systemic Treatment of Metastatic Clear Cell Renal Cell Carcinoma in 2018: Current Paradigms, Use of Immunotherapy, and Future Directions. European urology. 2019; 75: 100-10.

12. Motzer RJ, Rini BI, McDermott DF, Arén Frontera O, Hammers HJ, Carducci MA, et al. Nivolumab plus ipilimumab versus sunitinib in first-line treatment for advanced renal cell carcinoma: extended follow-up of efficacy and safety results from a randomised, controlled, phase 3 trial. The Lancet Oncology. 2019; 20: 1370-85.

13. Powles T, Rini B. Novel Agents and Drug Development Needs in Advanced Clear Cell Renal Cancer. Journal of clinical oncology : official journal of the American Society of Clinical Oncology. 2018: Jco2018792655.

14. Motzer RJ, Tannir NM, McDermott DF, Arén Frontera O, Melichar B, Choueiri TK, et al. Nivolumab plus Ipilimumab versus Sunitinib in Advanced Renal-Cell Carcinoma. The New England journal of medicine. 2018; 378: 1277-90

15. Cerezo M, Guemiri R, Druillennec S, Girault I, Malka-Mahieu H, Shen S, et al. Translational control of tumor immune escape via the eIF4F-STAT1-PD-L1 axis in melanoma. Nature medicine. 2018; 24: 1877-86.

16. Eichmüller SB, Osen W, Mandelboim O, Seliger B. Immune Modulatory microRNAs Involved in Tumor Attack and Tumor Immune Escape. Journal of the National Cancer Institute. 2017; 109: 10.

17. Ni X, Tao J, Barbi J, Chen Q, Park BV, Li Z, et al. YAP is essential for Treg mediated suppression of anti-tumor immunity. Cancer Discov. 2018; 8(8): 1026-1043. 
18. Groth $\mathrm{C}, \mathrm{Hu} \mathrm{X}$, Weber $\mathrm{R}$, Fleming $\mathrm{V}$, Altevogt $\mathrm{P}$, Utikal J, et al. Immunosuppression mediated by myeloid-derived suppressor cells (MDSCs) during tumour progression. Br J Cancer. 2019; 120(1): 16-25.

19. Chanmee T, Ontong P, Konno K, Itano N. Tumor-associated macrophages as major players in the tumor microenvironment. Cancers. 2014; 6: 1670-90.

20. Chong EA, Melenhorst JJ, Lacey SF, Ambrose DE, Gonzalez V, Levine BL, et al. PD-1 blockade modulates chimeric antigen receptor (CAR)-modified T cells: refueling the CAR. Blood. 2017; 129: 1039-41.

21. Ribas A, Lawrence D, Atkinson V, Agarwal S, Miller WH, Jr., Carlino MS, et al. Combined BRAF and MEK inhibition with PD-1 blockade immunotherapy in BRAF-mutant melanoma. Nature medicine. 2019; 25: 936-40.

22. Wang L, Saci A, Szabo PM, Chasalow SD, Castillo-Martin M, Domingo-Domenech J, et al. EMT- and stroma-related gene expression and resistance to PD-1 blockade in urothelial cancer. Nature communications. 2018; 9: 3503.

23. Neubert NJ, Schmittnaegel M, Bordry N, Nassiri S, Wald N, Martignier C, et al. $\mathrm{T}$ cell-induced CSF1 promotes melanoma resistance to PD1 blockade. Science translational medicine. 2018; 10

24. Belli C, Trapani D, Viale G, D'Amico P, Duso BA, Della Vigna P, et al. Targeting the microenvironment in solid tumors. Cancer treatment reviews. 2018; 65: 22-32.

25. Ren B, Cui M, Yang G, Wang H, Feng M, You L, et al. Tumor microenvironment participates in metastasis of pancreatic cancer. Mol Cancer. 2018; 17: 108.

26. Zizza P, Dinami R, Porru M, Cingolani C, Salvati E, Rizzo A, et al. TRF2 positively regulates SULF2 expression increasing VEGF-A release and activity in tumor microenvironment. Nucleic acids research. 2019; 47: 3365-82.

27. Quail DF, Joyce JA. Microenvironmental regulation of tumor progression and metastasis. Nature medicine. 2013; 19: 1423-37.

28. Hu G, Wu P, Cheng P, Zhang Z, Wang Z, Yu X, et al. Tumor-infiltrating CD39(+)yסTregs are novel immunosuppressive $T$ cells in human colorectal cancer. Oncoimmunology. 2017; 6: e1277305.

29. Triner D, Devenport SN, Ramakrishnan SK, Ma X, Frieler RA, Greenson JK, et al. Neutrophils Restrict Tumor-Associated Microbiota to Reduce Growth and Invasion of Colon Tumors in Mice. Gastroenterology. 2019; 156: 1467-82.

30. Wang Y, Wang Y, Xu L, Lu X, Fu D, Su J, et al. CD4 + T cells promote renal cell carcinoma proliferation via modulating YBX1. Experimental cell research. 2018; 363: 95-101.

31. Friedman J, Hastie T, Tibshirani R. Regularization Paths for Generalized Linear Models via Coordinate Descent. J Stat Softw. 2010; 33: 1-22.

32. Simon N, Friedman J, Hastie T, Tibshirani R. Regularization Paths for Cox's Proportional Hazards Model via Coordinate Descent. J Stat Softw. 2011; 39: 1-13.

33. Love MI, Huber W, Anders S. Moderated estimation of fold change and dispersion for RNA-seq data with DESeq2. Genome biology. 2014; 15: 550.

34. Yu G, Wang LG, Han Y, He QY. clusterProfiler: an R package for comparing biological themes among gene clusters. Omics : a journal of integrative biology. 2012; 16: 284-7.

35. Shannon P, Markiel A, Ozier O, Baliga NS, Wang JT, Ramage D, et al. Cytoscape: a software environment for integrated models of biomolecular interaction networks. Genome research. 2003; 13: 2498-504.

36. Bader GD, Hogue CW. An automated method for finding molecular complexes in large protein interaction networks. BMC bioinformatics. 2003; 4 : 2.

37. Subramanian A, Tamayo P, Mootha VK, Mukherjee S, Ebert BL, Gillette MA, et al. Gene set enrichment analysis: a knowledge-based approach for interpreting genome-wide expression profiles. Proceedings of the National Academy of Sciences of the United States of America. 2005; 102: 15545-50.

38. Mootha VK, Lindgren CM, Eriksson KF, Subramanian A, Sihag S, Lehar J, et al. PGC-1alpha-responsive genes involved in oxidative phosphorylation are coordinately downregulated in human diabetes. Nature genetics. 2003; 34: 267-73.

39. Wang $\mathrm{K}, \mathrm{Xu} \mathrm{T}$, Ruan H, Xiao H, Liu J, Song Z, et al. LXRa promotes cell metastasis by regulating the NLRP3 inflammasome in renal cell carcinoma. Cell death \& disease. 2019: 10: 159.

40. Weinstock M, McDermott D. Targeting PD-1/PD-L1 in the treatment of metastatic renal cell carcinoma. Therapeutic advances in urology. 2015; 7: 365-77.

41. Thomas JS, Kabbinavar F. Metastatic clear cell renal cell carcinoma: A review of current therapies and novel immunotherapies. Critical reviews in oncology/hematology. 2015; 96: 527-33.

42. Géczi L, Nagyiványi K, Maráz A. [Immunotherapy of renal cell cancer]. Magyar onkologia. 2017; 61: 126-31

43. Song C, Phuengkham H, Kim YS, Dinh VV, Lee I, Shin IW, et al. Syringeable immunotherapeutic nanogel reshapes tumor microenvironment and prevents tumor metastasis and recurrence. Nature communications. 2019; 10: 3745

44. Xiao Z, Dai Z, Locasale JW. Metabolic landscape of the tumor microenvironment at single cell resolution. Nature communications. 2019; 10: 3763.

45. Matoba T, Imai M, Ohkura N, Kawakita D, Ijichi K, Toyama T, et al. Regulatory $\mathrm{T}$ cells expressing abundant CTLA-4 on the cell surface with a proliferative gene profile are key features of human head and neck cancer. International journal of cancer. 2019; 144: 2811-22.

46. Kowanetz M, Zou W, Gettinger SN, Koeppen H, Kockx M, Schmid P, et al. Differential regulation of PD-L1 expression by immune and tumor cells in
NSCLC and the response to treatment with atezolizumab (anti-PD-L1). Proceedings of the National Academy of Sciences of the United States of America. 2018; 115: E10119-e26.

47. Shi C, Pamer EG. Monocyte recruitment during infection and inflammation. Nature reviews Immunology. 2011; 11: 762-74.

48. Liu LZ, Zhang Z, Zheng BH, Shi Y, Duan M, Ma LJ, et al. CCL15 Recruits Suppressive Monocytes to Facilitate Immune Escape and Disease Progression in Hepatocellular Carcinoma. Hepatology. 2019; 69: 143-59.

49. Qian BZ, Li J, Zhang H, Kitamura T, Zhang J, Campion LR, et al. CCL2 recruits inflammatory monocytes to facilitate breast-tumour metastasis. Nature. 2011; 475: 222-5

50. Lim SY, Yuzhalin AE, Gordon-Weeks AN, Muschel RJ. Tumor-infiltrating monocytes/macrophages promote tumor invasion and migration by upregulating S100A8 and S100A9 expression in cancer cells. Oncogene. 2016; 35: 5735-45.

51. Schmall A, Al-Tamari HM, Herold S, Kampschulte M, Weigert A, Wietelmann A, et al. Macrophage and cancer cell cross-talk via CCR2 and CX3CR1 is a fundamental mechanism driving lung cancer. American journal of respiratory and critical care medicine. 2015; 191: 437-47.

52. Hanna RN, Cekic C, Sag D, Tacke R, Thomas GD, Nowyhed H, et al. Patrolling monocytes control tumor metastasis to the lung. Science. 2015; 350: 985-90.

53. Kubo H, Mensurado S, Gonçalves-Sousa N, Serre K, Silva-Santos B. Primary Tumors Limit Metastasis Formation through Induction of IL15-Mediated Cross-Talk between Patrolling Monocytes and NK Cells. Cancer immunology research. 2017; 5: 812-20.

54. Romano E, Kusio-Kobialka M, Foukas PG, Baumgaertner P, Meyer C, Ballabeni $\mathrm{P}$, et al. Ipilimumab-dependent cell-mediated cytotoxicity of regulatory $\mathrm{T}$ cells $e x$ vivo by nonclassical monocytes in melanoma patients. Proceedings of the National Academy of Sciences of the United States of America. 2015; 112: 6140-5.

55. Gordon IO, Freedman RS. Defective antitumor function of monocyte-derived macrophages from epithelial ovarian cancer patients. Clinical cancer research : an official journal of the American Association for Cancer Research. 2006; 12: 1515-24

56. Yeap WH, Wong KL, Shimasaki N, Teo EC, Quek JK, Yong HX, et al. CD16 is indispensable for antibody-dependent cellular cytotoxicity by human monocytes. Scientific reports. 2016; 6: 34310.

57. Hannocks MJ, Zhang X, Gerwien $\mathrm{H}$, Chashchina A, Burmeister M, Korpos E, et al. The gelatinases, MMP-2 and MMP-9, as fine tuners of neuroinflammatory processes. Matrix biology : journal of the International Society for Matrix Biology. 2019; 75-76: 102-13.

58. Huang H. Matrix Metalloproteinase-9 (MMP-9) as a Cancer Biomarker and MMP-9 Biosensors: Recent Advances. Sensors (Basel, Switzerland). 2018; 18.

59. Chen YT, Yang CC, Shao PL, Huang CR, Yip HK. Melatonin-mediated downregulation of ZNF746 suppresses bladder tumorigenesis mainly through inhibiting the AKT-MMP-9 signaling pathway. Journal of pineal research. 2019; 66: e12536.

60. Liu L, Ye Y, Zhu X MMP-9 secreted by tumor associated macrophages promoted gastric cancer metastasis through a PI3K/AKT/Snail pathway. Biomedicine \& pharmacotherapy = Biomedecine \& pharmacotherapie. 2019; 117: 109096

61. Cheng Y, Cheng T, Zhao Y, Qu Y. HMGA1 exacerbates tumor progression by activating miR-222 through PI3K/Akt/MMP-9 signaling pathway in uveal melanoma. Cellular signalling. 2019; 63: 109386.

62. Osman M, Tortorella M, Londei M, Quaratino S. Expression of matrix metalloproteinases and tissue inhibitors of metalloproteinases define the migratory characteristics of human monocyte-derived dendritic cells. Immunology. 2002; 105: 73-82.

63. Sapharikas E, Lokajczyk A, Fischer AM, Boisson-Vidal C. Fucoidan Stimulates Monocyte Migration via ERK/p38 Signaling Pathways and MMP9 Secretion. Marine drugs. 2015; 13: 4156-70.

64. Zhou J, Zhang J, Chao J. Porphyromonas gingivalis promotes monocyte migration by activating MMP-9. Journal of periodontal research. 2012; 47: 236-42.

65. Kawai Y, Narita Y, Yamawaki-Ogata A, Usui A, Komori K. Montelukast, a Cysteinyl Leukotriene Receptor 1 Antagonist, Induces M2 Macrophage Polarization and Inhibits Murine Aortic Aneurysm Formation. BioMed research international. 2019; 2019: 9104680 .

66. Bae JH, Song DK, Im SS. Regulation of IGFBP-1 in Metabolic Diseases. Journal of lifestyle medicine. 2013; 3: 73-9.

67. Gupta MB. The role and regulation of IGFBP-1 phosphorylation in fetal growth restriction. Journal of cell communication and signaling. 2015; 9: $111-23$

68. Zheng $\mathrm{F}$, Tang $\mathrm{Q}$, Zheng $\mathrm{XH}$, Wu J, Huang $\mathrm{H}$, Zhang $\mathrm{H}$, et al. Inactivation of Stat3 and crosstalk of miRNA155-5p and FOXO3a contribute to the induction of IGFBP1 expression by beta-elemene in human lung cancer. Experimental \& molecular medicine. 2018; 50: 121

69. Kim JC, Ha YJ, Tak KH, Roh SA, Kim CW, Kim TW, et al. Complex Behavior of ALDH1A1 and IGFBP1 in Liver Metastasis from a Colorectal Cancer. PloS one. 2016; 11: e0155160.

70. Gong J, Shen S, Yang Y, Qin S, Huang L, Zhang H, et al. Inhibition of FASN suppresses migration, invasion and growth in hepatoma carcinoma cells by deregulating the HIF-1a/IGFBP1 pathway. International journal of oncology. 2017; 50: 883-92. 
71. Forbes BE, McCarthy $\mathrm{P}$, Norton RS. Insulin-like growth factor binding proteins: a structural perspective. Frontiers in endocrinology. 2012; 3: 38.

72. Brandt K, Grünler J, Brismar K, Wang J. Effects of IGFBP-1 and IGFBP-2 and their fragments on migration and IGF-induced proliferation of human dermal fibroblasts. Growth hormone \& IGF research : official journal of the Growth Hormone Research Society and the International IGF Research Society. 2015; 25: $34-40$.

73. Dorniak P, Bazer FW, Wu G, Spencer TE. Conceptus-derived prostaglandins regulate endometrial function in sheep. Biology of reproduction. 2012; 87(9): $1-7$.

74. Irving JA, Lala PK. Functional role of cell surface integrins on human trophoblast cell migration: regulation by TGF-beta, IGF-II, and IGFBP-1. Experimental cell research. 1995; 217: 419-27. 